\title{
A signature of tumor immune microenvironment genes associated with the prognosis of non-small cell lung cancer
}

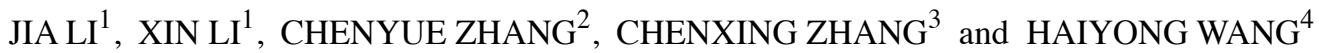 \\ ${ }^{1}$ Department of Oncology, Longhua Hospital, Shanghai University of Traditional Chinese Medicine; \\ ${ }^{2}$ Department of Integrative Oncology, Fudan University Shanghai Cancer Center, Shanghai 200032; \\ ${ }^{3}$ Department of Allergy and Immunology, Shanghai Children's Medical Center, Shanghai Jiao Tong University \\ School of Medicine, Shanghai 200127; ${ }^{4}$ Department of Internal Medicine-Oncology, \\ Shandong Cancer Hospital and Institute, Shandong First Medical University and \\ Shandong Academy of Medical Sciences, Jinan, Shandong 250117, P.R. China
}

Received July 30, 2019; Accepted December 9, 2019

DOI: $10.3892 /$ or.2020.7464

\begin{abstract}
Establishing a prognostic genetic signature closely related to the tumor immune microenvironment (TIME) to predict clinical outcomes is necessary. Using the Gene Expression Omnibus (GEO) database of a non-small cell lung cancer (NSCLC) cohort and the immune score derived from the Estimation of Stromal and Immune cells in Malignant Tumours using Expression data (ESTIMATE) algorithm, we applied the least absolute shrinkage and selection operator (LASSO) Cox
\end{abstract}

Correspondence to: Dr Haiyong Wang, Department of Internal Medicine-Oncology, Shandong Cancer Hospital and Institute, Shandong First Medical University and Shandong Academy of Medical Sciences, Jinan, Shandong 250117, P.R. China

E-mail: wanghaiyong6688@126.com

Abbreviations: TIME, tumor immune microenvironment; TCGA, The Cancer Genome Atlas; GEO, Gene Expression Omnibus; NSCLC, non-small cell lung cancer; ESTIMATE: Estimation of Stromal and Immune cells in Malignant Tumours using Expression data; LASSO, least absolute shrinkage and selection operator; TNM, Tumor, Lymph Node and Metastasis; NK, natural killer; OS, overall survival; ECM, extracellular matrix; EGFR, epidermal growth factor receptor; GO, Gene Ontology; DAVID, Database for Annotation, Visualization and Integrated Discovery; BP, biological processes; MF, molecular functions; CC, cellular components; KEGG, Kyoto Gene and Genomic Encyclopedia; LOOCV, leave-one-out cross-validation; ROC, receiver operating characteristic; AUC, area under the curve; NCAM2, neural cell adhesion molecule 2; NAV3, neuron navigator 3; $K D R$, kinase insert domain receptor; $I C A 1 L$, islet cell autoantigen 1-like; $C L N 5$, intracellular trafficking protein; ZBTB34, zinc finger and BTB domain containing 34; PRAM1, PML-RARA regulated adaptor molecule 1; TOMM6, translocase of outer mitochondrial membrane 6; ICI, immune checkpoint inhibitors; MCs, mast cells; CTL, cytotoxic T lymphocytes; MM, malignant melanoma

Key words: NSCLC, genetic signature, TIME, bioinformatics, prognosis regression model to screen a 10 -gene signature among the 448 differentially expressed genes and found that the risk prediction models constructed by 10 genes could be more sensitive to prognosis than TNM (Tumor, Lymph node and Metastasis) stage $(\mathrm{P}=0.006)$. The CIBERSORT method was applied to quantify the relative levels of different immune cell types. It was found that the ratio of eosinophils, mast cells (MCs) resting and CD4 T cells memory activated in the low-risk group was higher than that in the high-risk group, and the difference was statistically significant $(\mathrm{P}=0.003, \mathrm{P}=0.014$ and $\mathrm{P}=0.018$, respectively). Inconsistently, the ratio of resting natural killer (NK) cells and activated plasma cells in the low-risk group was significantly lower than that in the high-risk group $(\mathrm{P}=0.05$ and $\mathrm{P}=0.009$, respectively). Kaplan-Meier survival results showed that patients of the high-risk group had significantly shorter overall survival (OS) than those of the low-risk group in the training set $(\mathrm{P}<0.001)$. Furthermore, Kaplan-Meier survival showed that patients of the high-risk group had significantly shorter OS than those of the low-risk group $(\mathrm{P}=0.0025$ and $\mathrm{P}=0.0157$, respectively) in the validation set [GSE31210 and TCGA (The Cancer Genome Atlas)]. The 10-gene signature was found to be an independent risk factor for prognosis in univariate and multivariate Cox proportional hazard regression analyses $(\mathrm{P}<0.001)$. In addition, it was found that the risk model constructed by the 10-gene signature was related to the clinical related factors in logistic regression analysis. The genetic signature closely related to the immune microenvironment was found to be able to predict differences in the proportion of immune cells (eosinophils, resting MCs, memory activated CD4 T cells, resting NK cells and plasma cells) in the risk model. Our findings suggest that the genetic signature closely related to TIME could predict the prognosis of NSCLC patients, and provide some reference for immunotherapy.

\section{Introduction}

Lung cancer ranks first among all malignant tumors in regards to morbidity and mortality worldwide, with 2.1 million new lung cancer cases and 1.8 million deaths expected worldwide 
in 2018 (1). Non-small cell lung cancer (NSCLC) accounts for $85 \%$ of all lung cancer cases, and targeted therapy and immunotherapy for NSCLC treatment are developing rapidly $(2,3)$. Accurate judgment and prognostic assessment are important factors influencing the appropriate treatment for each individual case. The current TNM staging system has been tested over time and remains the most powerful prognostic instrument for lung cancer (4). However, due to the heterogeneity of the tumor itself and the complexity of the pathogenesis, even patients with the same TNM stage and treatment may exhibit various clinical outcomes (5). The current direction is to combine TNM with other prognostic factors to create a comprehensive prognostic indicator for NSCLC.

The tumor immune microenvironment (TIME) consists of immune cells, mesenchymal cells, endothelial cells, inflammatory mediators, and extracellular matrix (ECM) molecules $(6,7)$. The type, density and location of immune cells in TIME play an important role in the development of the disease and have been proposed to be valuable for the diagnosis and prognostic assessment of tumors (8). Therefore, immunological structures based on TIME should be used as a separate component in the classification system (9). Immunological analysis of the TIME (immunoscore) shows great promise for improved prognosis and prediction of response to immunotherapy. Several reports have demonstrated that immune scores and stromal scores calculated based on the ESTIMATE algorithm could predict the infiltration of non-tumor cells, by analyzing specific gene expression signature of immune and stromal cells (10-13).

For the first time in the present study, using the Gene Expression Omnibus (GEO) database of the NSCLC cohort and the immune score derived from the ESTIMATE algorithm, we extracted a genetic signature closely related to the TIME that predict the prognosis of lung cancer patients. Then the CIBERSORT method was used to quantify the relative levels of different immune cell types in complex gene expression mixtures. Furthermore, the validity and reliability of the gene signature were further verified. Our findings suggest that the genetic signature closely related to TIME is able to predict the prognosis of NSCLC patients, and provide some reference for immunotherapy.

\section{Materials and methods}

Data source and processing. Gene-expression profiling data of NSCLC patients were downloaded from Gene Expression Omnibus datasets (GEO; GSE103584 and GSE31210) and The Cancer Genome Atlas (TCGA; https://tcga-data.nci.nih. gov/tcga/). Microarray analysis of 130 NSCLC patients in GSE103584 was based on CancerSCAN panel (14). Dataset GSE103584 was used as a training set for model construction, and data in GSE31210 (15) and TCGA were applied to verify the validity of the model.

ESTIMATE algorithm-derived immune scores. Immune scores were calculated by applying the ESTIMATE algorithm using gene expression data.

The algorithm was publicly available through the SourceForge software repository (https://sourceforge. net/projects/estimateproject/) (13). The algorithm was based on single-sample gene set enrichment analysis and generates immune score (indicating the infiltration of immune cells in tumor tissue).

Differential gene screening related to immune scores and enrichment analysis of differentially expressed genes. The immune score for each sample in the training set was calculated according to the ESTIMATE algorithm, and the best cutoff value was generated using X-tile plots (16). Data analysis was performed using packaging limma (17). The relapse-free survival is defined as time from randomization to the first reucurrence or death. The overall survival is defined as the time from the initial confirmed diagnosis to the death of any cause. Fold change $>1.5$ and adj. $\mathrm{P}<0.05$ were set as the cutoff value for screening differentially expressed genes. First, the low immune score and high immune score samples were normalized by the limma package, and then the differential genes were screened to obtain 448 differentially expressed genes.

Functional enrichment analysis of the differential genes was performed using Database for Annotation, Visualization and Integrated Discovery (DAVID) (18) and GO categories were identified by their biological processes (BP), molecular functions (MF) and cellular components (CC). The DAVID database was also used for pathway enrichment analysis with reference to the Kyoto Encyclopedia of Gene and Genomic (KEGG) pathway. False discovery rate (FDR) $<0.05$ was used as the cutoff.

Screening for prognosis-related genes and building risk models. LASSO is a superior high-dimensional regression classifier and was used to select the key genes influencing patient outcomes (19). LASSO 1000 iterations were performed using the publicly available R package glmnet (20). Multiple genomes containing the optimal solution were received after multiple dimensionality reduction. At the same time, for the stability and accuracy of the results, a random sampling method of leave-one-out cross-validation (LOOCV) was used to select a set of genes to construct a prognostic model (19).

According to the selected genetic model, a risk formula of risk score was constructed to evaluate the high-risk and low-risk groups. The formula for obtaining the score is $\Sigma_{\mathrm{i}} \omega_{\mathrm{i}} \chi_{\mathrm{i}}$, where $\omega_{\mathrm{i}}$ and $\chi_{\mathrm{i}}$ are the coefficients and expressed value of each gene. The risk score for each sample in the data in the training set was calculated according to the formula, and the best cutoff value was generated using X-tile plots (16). This threshold was set to classify patients: Higher than the best cutoff for the low-risk group and lower than the risk score for the high-risk group.

Estimating the composition of immune cells. To estimate the immune cell composition in the sample, the analytical platform CIBERSORT (https://cibersort.stanford.edu/) was used to quantify the relative levels of distinct immune cell types within a complex gene expression mixture (21). The analysis was performed with an arrangement of 100 default statistical parameters. The activation and quiescence state of the same type of immune cells were analyzed as a whole. CIBERSORT's deconvolution of gene expression data provides valuable information about the composition of immune cells in a sample.

Validation of the validity and reliability. Univariate survival analysis of the gene signature was assessed by using survival 
in $\mathrm{R}$ language $(\mathrm{P}<0.05)(22)$. Then survival receiver operating characteristic curve (ROC) was used to complete the area under the curve (AUC) of gene signature and TNM classification (23). External data from GSE31210 and TCGA were applied to verify the reliability of the gene signature's impact on the prognosis of the patients.

The univariate and multivariate Cox proportional hazard regression analyses were used to evaluate independent prognostic factors associated with survival. Gene signature, age, sex, smoking status, T stage T, N stage, histology, grade, epidermal growth factor receptor (EGFR) mutation status and adjuvant chemotherapy were employed as covariates. In addition, the logistic regression analysis were used to analyze the association between the clinical related factors and risk model of gene signature construction.

\section{Results}

Correlation between immune score and overall survival in patients with NSCLC. There were 13,035 gene expression profiles obtained from 130 tumor samples in the dataset GSE103584 (Table SI). After normalizing the data of 130 samples, the immune score, stromal score, and estimate score were calculated by immunocyte-related genes (Table SII). The 21 low immune score samples and 109 high immune score samples were divided by X-tile plots (Fig. $1 \mathrm{~A}$ and $\mathrm{B}, \mathrm{P}<0.001$ ). Kaplan-Meier survival curves showed that the relapse-free survival of patients with the high score group of immune scores was longer than that when compared with the patients in the low score group (Fig. $1 \mathrm{C}, \mathrm{P}<0.001$ ). Consistently, patients with high immune scores also showed longer overall survival compared to the patients with low scores (Fig. 1D, $\mathrm{P}=0.017$ ).

Differential gene screening related to immune scores and enrichment analysis of the differential genes. To reveal the correlation of gene expression profiles with immune scores, we compared the gene microarray data of all 130 cases obtained in the dataset GSE103584. The low immune score and high immune score samples were normalized by the limma package and 448 differential genes were extracted from the comparison of high vs. low immune score groups. Heatmaps showed distinct gene expression profiles of cases in the low vs. high low immune score groups (Fig. 2A).

To analyze the potential functions of differential genes, we performed functional enrichment analysis on 448 differentially expressed genes. The Gene Ontology (GO) terminology was identified. The top 10 positions of the GO term for biological processes, cellular component terms and molecular functions are listed (Fig. 2B). The top GO terms included 'extracellular matrix organization' and 'extracellular structure organization' closely related to the immune microenvironment of tumors. In addition, pathway enrichment analysis with reference to KEGG mainly focused on 'protein digestion and absorption', 'AGE-RAGE signaling pathway in diabetic complications', 'platelet activation and focal adhesion' which also had a relationship with immune response (Fig. 2C).

Screening genes associated with prognosis and building risk models. We applied the LASSO Cox regression model to predict and analyze the most valuable prognostic genes among the 448 differential genes in the 130 sample data. A random sampling method of 10-cross validation was used to construct a prognostic model containing 10 genes (Fig. 3A). Through calculation and verification, it was found that the model constructed by 10 genes had the lowest error rate (Fig. 3B). Fig. $3 \mathrm{C}$ shows the specific information and coefficients of the 10 genes.

To further validate the accuracy of the risk prediction model, we established a ROC plot of the signature model and TNM stage. As shown in Fig. 3D, we found that risk prediction models were more sensitive to prognosis than the TNM stage $(\mathrm{P}=0.006)$.

Estimating the composition of immune cells. We used CIBERSORT to estimate the immune cell composition of the 130 samples and quantify the relative levels of different cell types in a mixed cell population. All results were normalized relative proportions by cell type (Table SIII). As shown in Fig. 4A, B and E, we compared different types of cells in the low-risk group and the high-risk group. It was found that the ratio of eosinophils, mast cells resting and CD4 T cells memory activated in the low-risk group was higher than that in the high-risk group, and the difference was statistically significant $(\mathrm{P}=0.003, \mathrm{P}=0.014$ and $\mathrm{P}=0.018$, respectively). Inconsistently, the ratio of $\mathrm{NK}$ cells resting and plasma cells activated in the low-risk group was lower than that in the high-risk group $(\mathrm{P}=0.05$ and $\mathrm{P}=0.009$, respectively) (Fig. 4C and D). The results indicated that activation and inhibition of various immune cells existed simultaneously in the tumor microenvironment.

Validation of the validity and reliability. Survival analysis in $\mathrm{R}$ language package was applied to examine the effects of the 10-gene signature on the prognosis of NSCLC patients. Kaplan-Meier survival curves for overall survival were used to represent the survival probabilities of the high-risk group and the low-risk group. The results showed that patients in the high-risk group had shorter overall survival than patients in the low-risk group (Fig. 5A, $\mathrm{P}<0.001$ ).

Furthermore, external data from GSE31210 and TCGA were applied as a validating set to verify the validity and reliability of the 10-gene signature impact on the prognosis of the NSCLC patients. Kaplan-Meier survival showed that patients in the high-risk group had shorter overall survival than patients in the low-risk group (Fig. 5B, $\mathrm{P}=0.0025$ and Fig. 5C, $\mathrm{P}=0.0157$ ).

Correlation of the clinical information. The correlation analysis between gene signature and clinical pathological parameters in the training set (GSE103584) is shown in Table I. The high-risk group was found to be significantly associated with all clinical pathological parameters. The univariate and multivariate Cox proportional hazard regression analyses were used to evaluate independent prognostic factors associated with survival. Gene signature, age, sex, smoking status, T stage, $\mathrm{N}$ stage $\mathrm{N}$, histology, grade, EGFR mutation status and adjuvant chemotherapy were employed as covariates. It was found that the risk model constructed by the 10 -gene signature was an independent risk factor for prognosis (Table II, $\mathrm{P}<0.001$ ). 


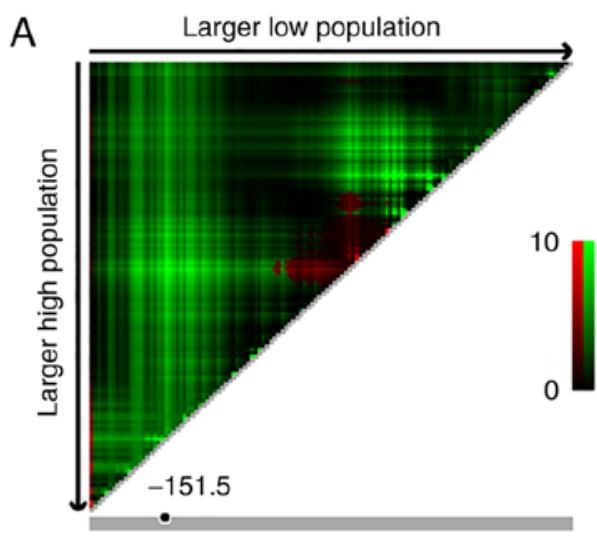

C

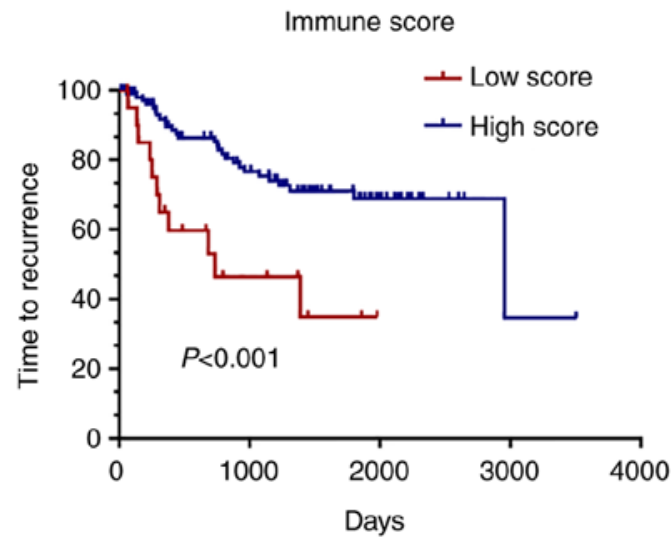

B

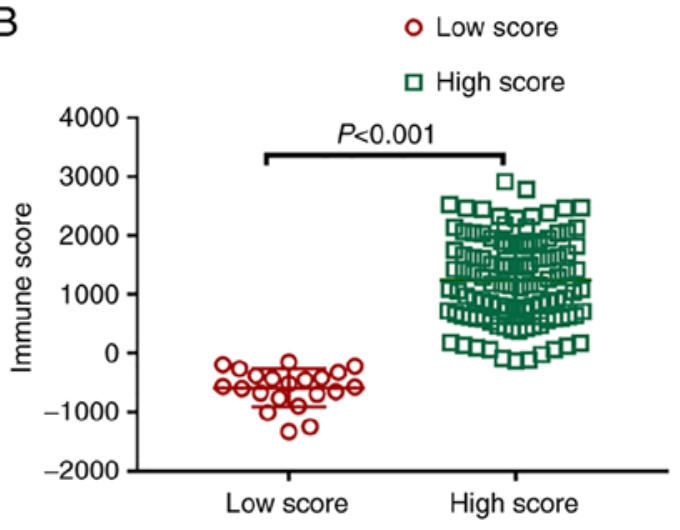

$\mathrm{D}$

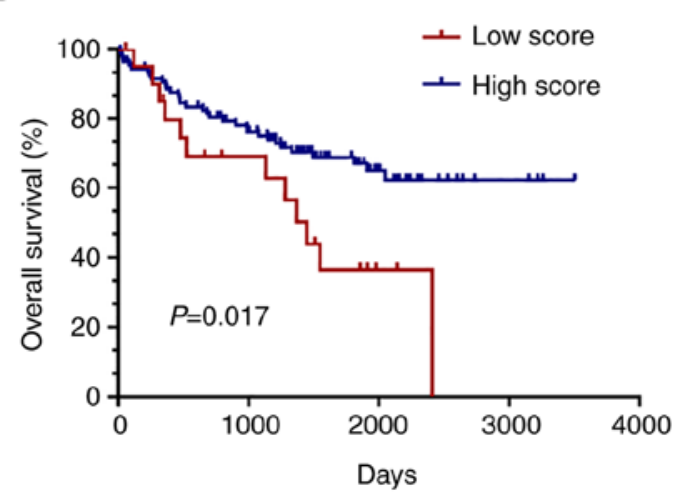

Figure 1. Correlation between immune score and overall survival in patients with NSCLC. (A) X-tile plot pattern diagram. The red portion represent samples with a low immune score and the green portion represent samples with a high immune score. -151.5 is the AQUA score for this set of samples to determine the best cutoff value by X-title software. 0-10 represents the level of expression intensity. (B) Comparison of immune scores between low-immunity score samples and high-immunity score samples. (C) Kaplan-Meier survival curves for relapse-free survival in the immune score groups. (D) Kaplan-Meier survival curves for overall survival in the immune score groups. NSCLC, non-small cell lung cancer.

In addition, logistic regression analysis was used to analyze the association between the clinical related factors and risk model of 10 -gene signature construction. As shown in Table III, we analyzed the risk factors for the risk model of the 10-gene signature construction. Age, sex, smoking status, $\mathrm{T}$ stage, $\mathrm{N}$ stage, histology, grade, EGFR mutation status and adjuvant chemotherapy were selected in the logistic regression model. $\mathrm{T}$ stage, $\mathrm{N}$ stage and EGFR mutation status were all independent risk factors in the multivariable analysis. The results showed that the patients with Stage T2 and Stage T4 had a significantly higher risk than those with Stage T0-1 (OR 3.822, 95\% CI 1.422-10.269, $\mathrm{P}=0.008$; OR 19.671, 95\% CI 2.304-167.949, $\mathrm{P}=0.006$ ) and the patients with Stage N2 had a significantly higher risk than those with Stage N0 (OR 13.066, 95\% CI 2.680-63.700 P=0.001). We also found that the patients without EGFR mutations had a significantly higher risk than those with EGFR mutations (OR 16.150,95\% CI 2.122-122.877, $\mathrm{P}=0.007)$.

\section{Discussion}

Malignant solid tumor tissues include not only tumor cells, but also tumor-associated normal epithelial cells and stromal cells, immune cells and vascular cells. Infiltrating immune cells are an integral component of the tumor immune microenvironment
(TIME) and play an important role in increasing the effectiveness of immunotherapy (24). This infiltrating immune cell population is usually a heterogeneous mixture of immune cells, including cell types associated with activity and inhibition (25). Because of the need for different types and subtypes of TIME to be identified in the immunotherapy of tumors, their characteristics and differences must be identified (26). In order to ensure substantial progress, bioinformatic techniques are used to assess the composition, functional status and cellular localization of immune cells. Based on gene signature, a more precise classification of patients based on their TIME will better predict overall survival and response to immunotherapeutic agents.

Firstly, we utilized an ESTIMATE algorithm to calculate immune scores and predict the level of infiltrating immune cells by immunocyte-related genes. The 21 low immune score samples and 109 high immune score samples were divided by X-tile plots. Kaplan-Meier survival curves showed that relapse-free survival and overall survival of patients in the high score group of immune scores was longer than the patients in the low score group. Next, 448 differential genes were extracted from the comparison of high vs. low immune score groups and the top Gene Ontology (GO) terms included extracellular matrix organization and extracellular structure organization closely related to the immune microenvironment 

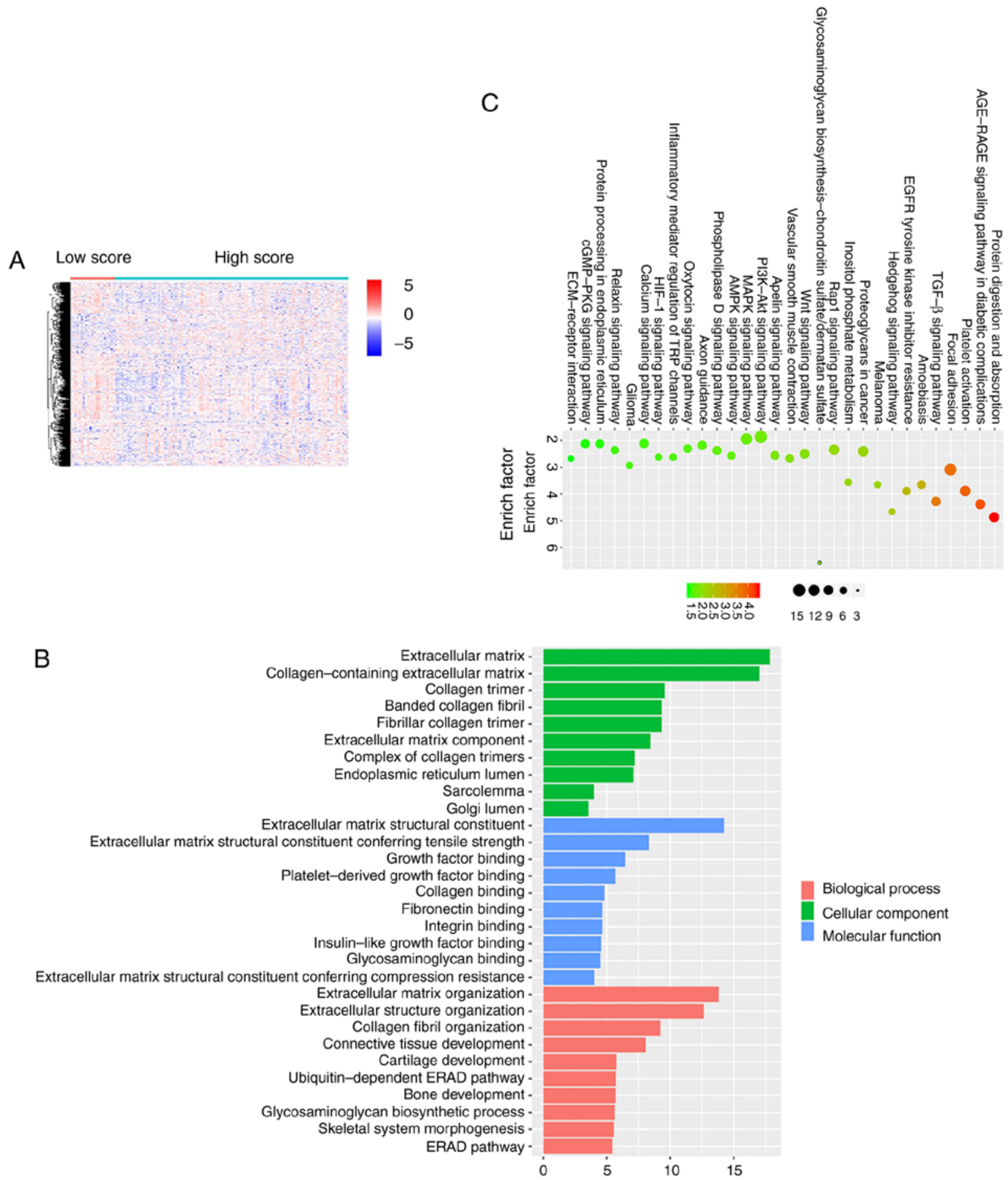

Figure 2. Differential gene screening related to immune scores and enrichment analysis of differential genes. (A) Heatmaps of the differential gene expression profile in the low immune score group and the high immune score group. Color represents the level of gene expression: Red represents high expression, and blue represents low expression. -5 stands for the lowest and 5 stands for the highest. (B) The top 10 positions of the Gene Ontology (GO) terminology for biological processes, cellular component terms and molecular functions. (C) The KEGG pathway enrichment analysis. Blue represents less enrichment to a pathway factor, and red represents more factors that are enriched into a pathway. Black dots represent the number of enrichment factors, the larger the dot, the higher the number. KEGG, Kyoto Gene and Genomic Encyclopedia.

of tumors. In addition, pathway enrichment analysis with reference to KEGG mainly focused on 'protein digestion and absorption', 'AGE-RAGE signaling pathway in diabetic complications', 'platelet activation and focal adhesion' which also had a relationship with immune response.

Furthermore, we applied the LASSO Cox regression model to screen a 10 -gene signature among the 448 differential genes and found that risk prediction models constructed by 10 genes were more sensitive to prognosis than TNM stage. That is the 10 differential gene signature including neural cell adhesion molecule 2 (NCAM2), caldesmon 1 (CALD1), neuron navigator 3 (NAV3), kinase insert domain receptor $(K D R)$, islet cell autoantigen 1-like (ICAIL), CLN5 intracellular trafficking protein (CLN5), zinc finger and BTB domain 

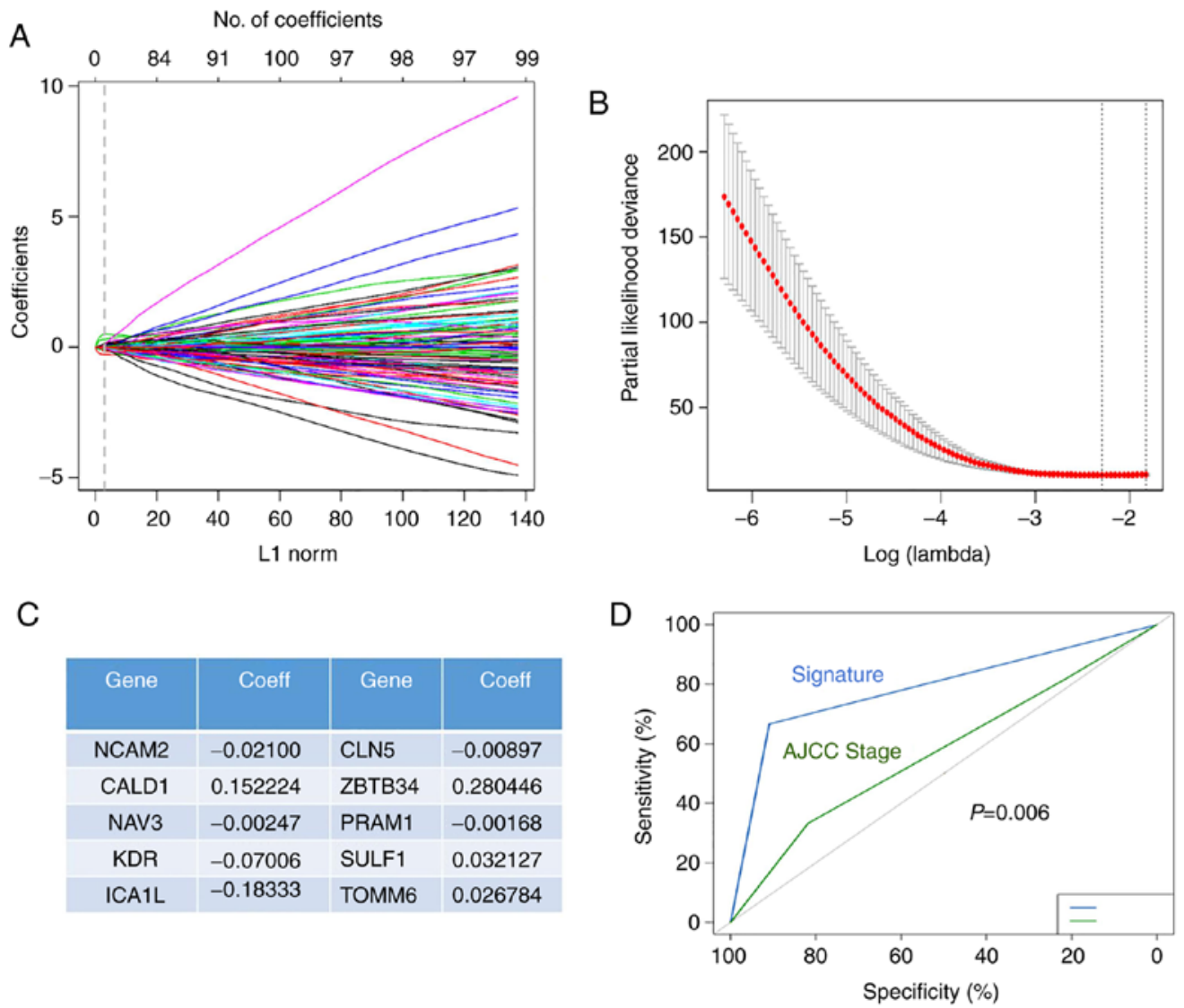

Figure 3. Screening genes associated with prognosis and building risk models. (A) Trend graph of LASSO coefficients. The lines represent the coefficient of Lasso. L1 norm represents the calculation method of random sampling method. (B) Partial likehood deviation map. (C) The name and coefficient of the 10-gene signature closely related to TIME. The partial likelihood represents the error rate chosen by random sampling. (D) ROC curves of the risk model and TNM staging in the training set. TIME, tumor immune microenvironment; LASSO, least absolute shrinkage and selection operator; ROC, receiver operating characteristic; $N C A M 2$, neural cell adhesion molecule $2 ; N A V 3$, neuron navigator $3 ; K D R$, kinase insert domain receptor; ICA1L, islet cell autoantigen 1-like; CLN5, intracellular trafficking protein; ZBTB34, zinc finger and BTB domain containing 34; PRAM1, PML-RARA regulated adaptor molecule 1; TOMM6, translocase of outer mitochondrial membrane 6; CALD1, caldesmon 1; SULF1, sulfatase 1.

containing 34 (ZBTB34), PML-RARA regulated adaptor molecule 1 (PRAM1), sulfatase 1 (SULF1) and translocase of outer mitochondrial membrane 6 (TOMM6) may influence the survival time of NSCLC patients.

Moreover, survival analysis in $\mathrm{R}$ language pack was applied to examine the effects of the 10-gene signature on the prognosis of NSCLC patients. Kaplan-Meier survival curves for overall survival showed that patients in the high-risk group had shorter over survival than patients in the low-risk group. Then, external data from GSE31210 and The Cancer Genome Atlas (TCGA) were applied as a validating set to verify the validity and reliability of the 10-gene signature impact on the prognosis of the patients. Kaplan-Meier survival showed that indeed patients in the high-risk group had shorter overall survival than patients in the low-risk group.

We not only confirmed the stability and accuracy of the 10 -gene signature, but also found it was closely associated with other clinical information. The univariate and multivariate Cox proportional hazard regression analyses were used to evaluate independent prognostic factors associated with survival. It was found that the risk model constructed by the 10 -gene signature was an independent risk factor for prognosis (Table III). In addition, logistic regression analysis was used to analyze the association between the clinical related factors and risk model of 10-gene signature construction. T stage, $\mathrm{N}$ stage and EGFR mutation status were all independent risk factors in the multivariable analysis. The results showed that the patients with Stage T2 and T4 had a significantly higher risk than those with Stage T0-1 and the patients with Stage N2 had a significantly higher risk than those with Stage N0. We also found that the patients without EGFR mutations had a significantly higher risk than those with EGFR mutations. These results suggest that our characteristics may contribute to the clinical management of NSCLC.

Finally, we used CIBERSORT to estimate the immune cell composition of 130 samples and quantify the relative levels of the different cell types in a mixed cell population and compared the different types of cells in the low-risk group and the high-risk group. Surprisingly, it was found that the ratio of eosinophils, mast cells resting and CD4 T cells memory activated in the low-risk group was higher than that in the high-risk group, and the difference was statistically significant. Inconsistently, the ratio of NK cells resting and plasma cells activated in the low-risk group was lower than that in the high-risk group. The results indicated that activation and inhibition of various immune cells existed simultaneously in TIME. The 10-gene signature was used to analyze the composition of immune cells helping to clarify the role of TIME 
A

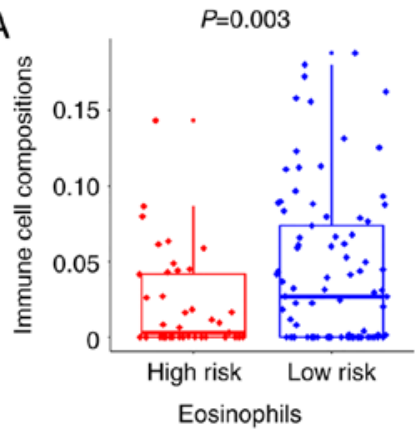

B

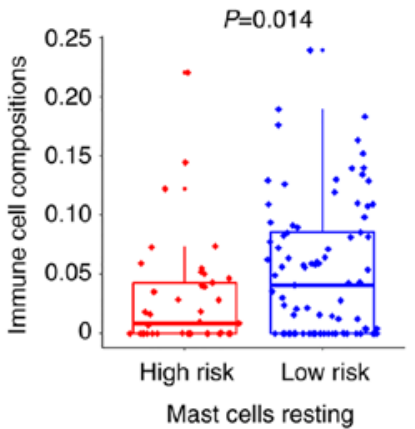

C

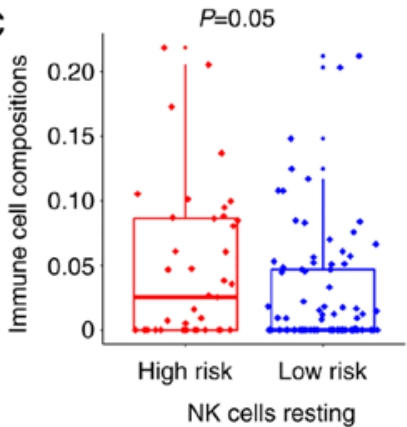

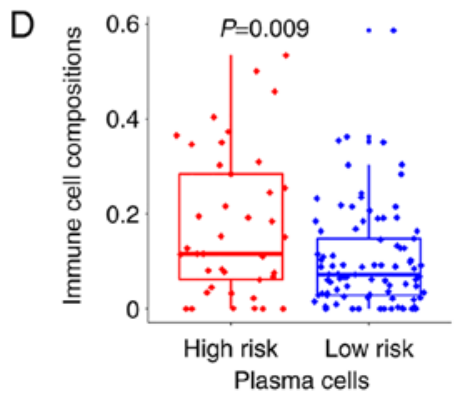

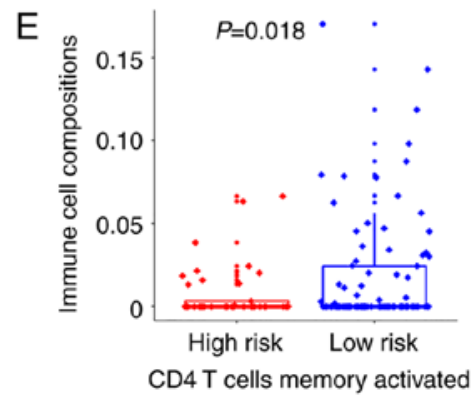

Figure 4. Estimating the composition of immune cells. (A) The ratio of eosinophils activated in the high-risk and low-risk group. (B) The ratio of mast cells resting in the high-risk and low-risk group. (C) The ratio of NK cells resting in the high-risk and low-risk group. (D) The ratio of plasma cells in the high-risk and low-risk group. (E) The ratio of CD4 T cells memory activated in the high-risk and low-risk group. NK, natural killer.
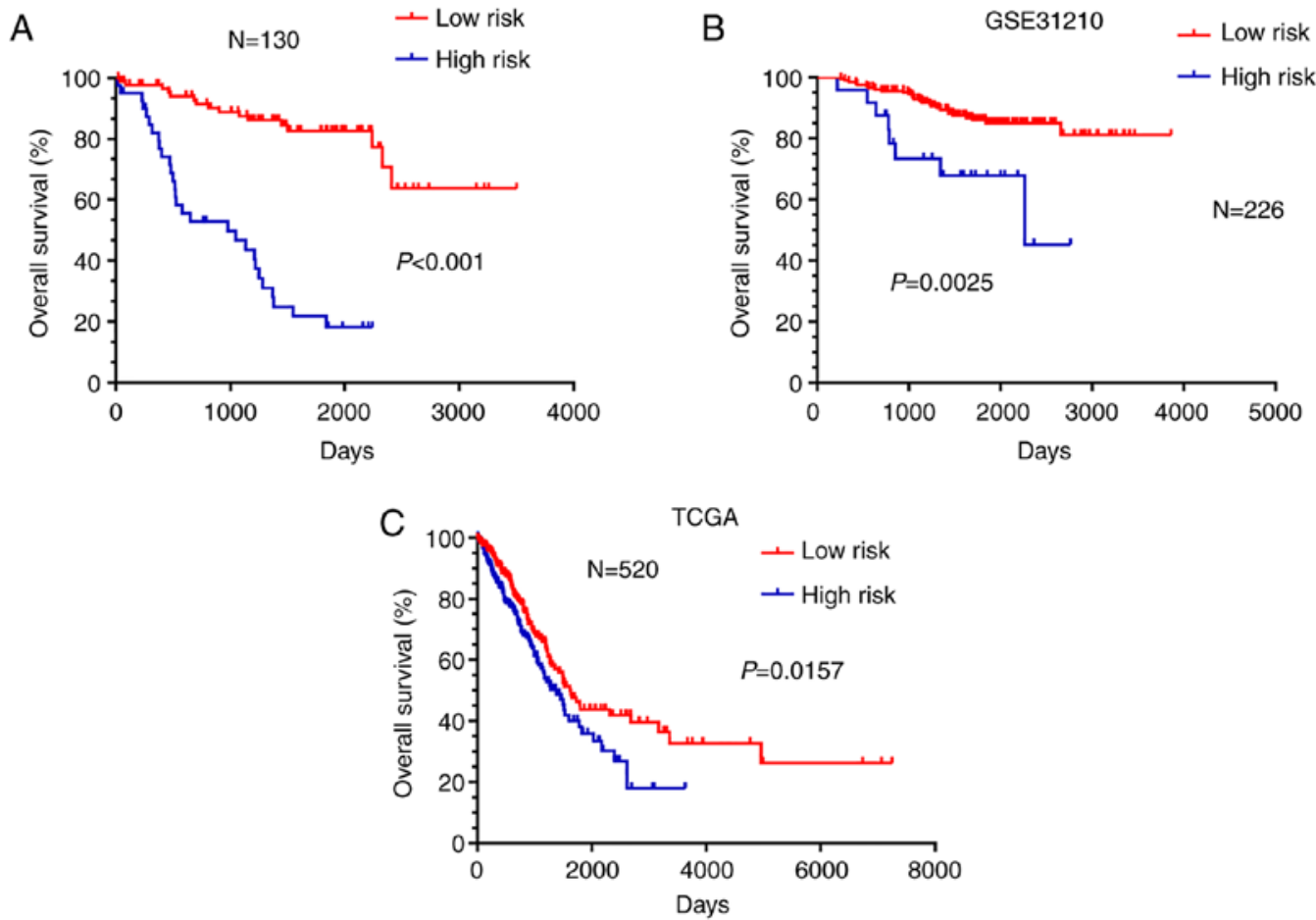

Figure 5. Kaplan-Meier survival curves for overall survival in the training and validating set. (A) Kaplan-Meier survival curves for overall survival in the training set. (B) Kaplan-Meier survival curves for overall survival in the GSE31210 set. (C) Kaplan-Meier survival curves for overall survival in TCGA. TCGA, The Cancer Genome Atlas.

and increase our understanding of molecular phenotype. The success of cancer immunotherapy has revolutionized cancer treatment and has used TIME parameters (immune cell composition and proportion) as predictive immunotherapy markers (8). Detailed characterization of the immune cell composition in tumors may be the basis for determining the prognostic and predictive biomarkers of immunotherapy $(27,28)$. Therefore, incorporating TIME parameters into a gene signature can be more conducive to individualized treatment options (29). Studies have reported that the expression levels of various proliferation-related genes are related to the response to immune checkpoint inhibitors in NSCLC (30). 
Table I. Correlation analysis between genetic signature and clinical pathological parameters of the NSCLC patients in the training set (GSE103584).

\begin{tabular}{|c|c|c|c|}
\hline Variables & $\begin{array}{c}\text { Low risk } \\
\mathrm{n}(\%)\end{array}$ & $\begin{array}{c}\text { High risk } \\
\mathrm{n}(\%)\end{array}$ & P-value \\
\hline Age (years) & & & $<0.001$ \\
\hline$<65$ & $29(32.6)$ & $8(19.5)$ & \\
\hline$>64$ & $60(67.4)$ & $33(80.5)$ & \\
\hline Sex & & & $<0.001$ \\
\hline Female & $26(29.2)$ & $8(19.5)$ & \\
\hline Male & $63(70.8)$ & $33(80.5)$ & \\
\hline Smoking status & & & $<0.001$ \\
\hline Yes & $75(84.3)$ & $35(85.4)$ & \\
\hline No & $14(15.7)$ & $6(14.6)$ & \\
\hline T stage & & & $<0.001$ \\
\hline $\mathrm{T} 0-1$ & $46(51.7)$ & $12(29.3)$ & \\
\hline $\mathrm{T} 2$ & $28(31.5)$ & $21(51.2)$ & \\
\hline $\mathrm{T} 3$ & $13(14.6)$ & $3(7.3)$ & \\
\hline $\mathrm{T} 4$ & $2(2.2)$ & $5(12.2)$ & \\
\hline $\mathrm{N}$ stage & & & $<0.001$ \\
\hline NO & $76(85.4)$ & $28(68.3)$ & \\
\hline N1 & $9(10.1)$ & $3(7.3)$ & \\
\hline $\mathrm{N} 2$ & $4(4.5)$ & $10(24.4)$ & \\
\hline Histology & & & $<0.001$ \\
\hline Adenocarcinoma & $67(75.3)$ & $29(70.7)$ & \\
\hline Squamous & $21(23.6)$ & $10(24.4)$ & \\
\hline Others & $1(1.1)$ & $2(4.9)$ & \\
\hline Grade & & & $<0.001$ \\
\hline I & $17(19.1)$ & $5(12.2)$ & \\
\hline II & $44(49.4)$ & $18(43.9)$ & \\
\hline III & $16(20.0)$ & $11(26.8)$ & \\
\hline Other & $12(13.5)$ & $7(17.1)$ & \\
\hline EGFR status & & & $<0.001$ \\
\hline Yes & $17(19.1)$ & $2(4.9)$ & \\
\hline No & $49(55.1)$ & $33(80.5)$ & \\
\hline Unknown & $23(25.8)$ & $6(14.6)$ & \\
\hline Adjuvant therapy & & & $<0.001$ \\
\hline Yes & $66(74.2)$ & $14(34.1)$ & \\
\hline No & $23(25.8)$ & $27(65.9)$ & \\
\hline
\end{tabular}

NSCLC, non-small cell lung cancer; EGFR, epidermal growth factor receptor.

In addition, JAK1/2 mutations are associated with resistance to anti-PD-1/PD-L1 antibodies and MDM2/MDM4 and EGFR changes may be associated with hyperprogression (31-33). Here, the 10-gene signature closely related to TIME could predict the prognosis of lung cancer patients, and provide some reference for immunotherapy.

Notably, among the 10-gene signature, only the gene $K D R$ is involved in tumor immunity and most of the genes (NCAM2, CALD1, NAV3, PRAM-1 and SULF1) are closely related to tumors and can be used as novel tumor biomarkers. There are also two other genes (ICAIL, CLN5) that have been reported to have related functions, but no reports exist on tumors and immunity. The TOMM6 gene has not been reported. NCAM2 is a close homolog of the neuronal cell adhesion molecule NCAM1 and stimulates neurite outgrowth through FGFR-dependent activation of the Ras/MAPK pathway $(34,35)$. Several studies have reported that NCAM2 can be used as a new therapeutic target for cancer, especially prostate cancer and breast cancer (36-38). CALD1 is a novel target of the TEA domain family member (39). Moreover, $C A L D 1$ encodes the caldesmon protein, which is a calmodulin-binding and cytoskeleton-associated protein and regulates cell motility, such as migration and invasion $(40,41)$. It has been suggested that CALD1 may indicate a general splicing event associated with cancer $(41,42)$ and was also identified as a potential prognostic molecular marker for bladder and colon cancer (43-45).

$N A V 3$ is a novel cancer-associated gene located at chromosome 12q21 and belongs to the 'hill' genes of genomic landscaping associated with cancer (46). Accumulating evidence suggests that the NAV3 gene is a key player in a variety of cancers, with downregulation of $N A V 3$ found in $40 \%$ of primary neuroblastomas and adrenocortical carcinomas $(47,48)$. NAV3 mutations have been found in melanoma, pancreatic cancer, breast cancer and colon cancer (49). We also found $N A V 3$ gene copy number changes (deletions/amplifications) in other cancer types of epithelial origin (50) and $N A V 3$ gene allelic loss was found to be associated with several subtypes of cutaneous T-cell lymphoma $(51,52)$. KDR, also known as VEGFR2, is expressed in endothelial cells (ECs) to promote EC growth and survival, thereby initiating angiogenesis (53). Research has shown that T cell KDR is an important molecule in immunity, and it was found that KDR was induced to be expressed in activated CD4 and CD8 T cells in vitro (54). In addition, KDR was also demonstrated to be expressed on $\mathrm{T}$ cells after interaction with tumor necrosis factor (TNF)-activated ECs, and have a function in transendothelial migration (55).

$I C A I L$ is highly expressed in sperm cells and is closely related to male infertility (56). CLN5 mutations cause neurodegenerative diseases, and symptoms include mainly seizures, visual failure, motor decline, and progressive cognitive deterioration (57). ZBTB34 encodes a nuclear protein and functions as a potential transcriptional repressor. The transcript of ZBTB34 appears in a variety of adult tissues related to the immune, nervous, muscle and endocrine systems suggesting that ZBTB34 is a ubiquitously expressed protein that may function universally in transcriptional regulation (58). PRAM-1 is an intracellular adaptor molecule that is upregulated during the induced granulocytic differentiation of promyelocytic leukemic cells and during normal human myelopoiesis (59). PRAM-1 is involved in a signaling pathway induced by retinoic acid in acute promyelocytic leukemia (APL) cells (60). SULF1 plays a key role in the pathogenesis of various types of human cancer, and SULF1 protein is secreted to the cell surface to regulate the sulfation of heparan sulfate proteoglycans (HSPGs) $(61,62)$. SULF1 was also found to be a novel prognostic marker and predictor of lymph node metastasis in patients with 
Table II. Univariate and multivariate Cox proportional hazard regression analyses between the risk factors and overall survival of NSCLC patients.

\begin{tabular}{|c|c|c|c|c|}
\hline \multirow[b]{2}{*}{ Variables } & \multicolumn{2}{|c|}{ Univariate analysis } & \multicolumn{2}{|c|}{ Multivariate analysis } \\
\hline & Wald $\chi^{2}$ & P-value & $\mathrm{HR}(95 \% \mathrm{CI})$ & P-value \\
\hline $\begin{array}{l}\text { Age (years) } \\
<65 \\
>64\end{array}$ & 0.253 & 0.615 & & NI \\
\hline $\begin{array}{l}\text { Sex } \\
\text { Female } \\
\text { Male }\end{array}$ & 0.432 & 0.511 & & NI \\
\hline $\begin{array}{l}\text { Smoking status } \\
\text { Yes } \\
\text { No }\end{array}$ & 0.443 & 0.506 & & NI \\
\hline $\begin{array}{l}\text { T stage } \\
\text { T0-1 } \\
\text { T2 } \\
\text { T3 } \\
\text { T4 }\end{array}$ & 2.545 & 0.467 & & NI \\
\hline $\begin{array}{l}\text { N stage } \\
\text { N0 } \\
\text { N1 } \\
\text { N2 }\end{array}$ & 4.706 & 0.095 & & NI \\
\hline $\begin{array}{l}\text { Histology } \\
\text { Adenocarcinoma } \\
\text { Squamous } \\
\text { Others }\end{array}$ & 0.379 & 0.827 & & NI \\
\hline $\begin{array}{l}\text { Grade } \\
\text { I } \\
\text { II } \\
\text { III } \\
\text { Other }\end{array}$ & 3.066 & 0.382 & & NI \\
\hline $\begin{array}{l}\text { EGFR status } \\
\text { Yes } \\
\text { No } \\
\text { Unknown }\end{array}$ & 0.975 & 0.614 & & NI \\
\hline $\begin{array}{l}\text { Adjuvant therapy } \\
\text { Yes } \\
\text { No }\end{array}$ & 0.766 & 0.381 & & NI \\
\hline $\begin{array}{l}\text { Gene signature } \\
\text { Low risk }\end{array}$ & 26.149 & $<0.001$ & Reference & $<0.001$ \\
\hline High risk & & & $8.828(3.831-20.342)$ & $<0.001$ \\
\hline
\end{tabular}

NSCLC, non-small cell lung cancer; EGFR, epidermal growth factor receptor; CI, confidence interval; NI, not included.

gastric cancer (63). From the above results, we can conclude that our gene signature not only identified new promising biomarkers, but also may provide a direction for the study of TIME mechanisms.

In summary, the present research has the following novelty and innovation. First, multiple bioinformatic analysis methods were used to extracted a 10-gene signature closely related to TIME. Second, the risk model constructed by the 10-gene signature was able to predict the prognosis of NSCLC patients and was more sensitive for predicting prognosis than TNM stage. Third, the 10-gene signature was found to be closely related to TIME parameters (immune cell composition and proportion) and provides a certain reference for the immunotherapy of NSCLC. Fourth, some previously 
Table III. Logistic regression analysis between the clinical related factors and risk model of genetic signature construction $(\mathrm{HR}=1)$.

\begin{tabular}{|c|c|c|c|c|}
\hline \multirow[b]{2}{*}{ Variables } & \multicolumn{2}{|c|}{ Univariate analysis } & \multicolumn{2}{|c|}{ Multivariate analysis } \\
\hline & Wald $\chi^{2}$ & P-value & OR $(95 \% \mathrm{CI})$ & P-value \\
\hline $\begin{array}{l}\text { Age (years) } \\
<65 \\
>64\end{array}$ & 1.010 & 0.315 & & NI \\
\hline $\begin{array}{l}\text { Sex } \\
\text { Female } \\
\text { Male }\end{array}$ & 1.656 & 0.198 & & NI \\
\hline $\begin{array}{l}\text { Smoking status } \\
\text { Yes } \\
\text { No }\end{array}$ & 0.478 & 0.490 & & NI \\
\hline $\begin{array}{l}\text { T stage } \\
\text { T0-1 }\end{array}$ & 10.720 & 0.013 & Reference & 0.009 \\
\hline $\mathrm{T} 2$ & & & $3.822(1.422-10.269)$ & 0.008 \\
\hline $\mathrm{T} 3$ & & & $1.218(0.255-5.823)$ & 0.805 \\
\hline $\mathrm{T} 4$ & & & 19.671 (2.304-167.949) & 0.006 \\
\hline $\begin{array}{l}\text { N stage } \\
\text { N0 }\end{array}$ & 8.652 & 0.013 & Reference & 0.006 \\
\hline N1 & & & $1.042(0.229-4.750)$ & 0.957 \\
\hline $\mathrm{N} 2$ & & & $13.066(2.680-63.700)$ & 0.001 \\
\hline $\begin{array}{l}\text { Histology } \\
\text { Adenocarcinoma } \\
\text { Squamous } \\
\text { Others }\end{array}$ & 0.102 & 0.950 & & NI \\
\hline $\begin{array}{l}\text { Grade } \\
\text { I } \\
\text { II } \\
\text { III } \\
\text { Others }\end{array}$ & 0.867 & 0.833 & & NI \\
\hline $\begin{array}{l}\text { EGFR status } \\
\text { Yes }\end{array}$ & 7.980 & 0.018 & Reference & 0.010 \\
\hline $\begin{array}{l}\text { No } \\
\text { Unknown }\end{array}$ & & & $\begin{array}{c}16.150(2.122-122.877) \\
5.985(0.688-52.088)\end{array}$ & $\begin{array}{l}0.007 \\
0.105\end{array}$ \\
\hline $\begin{array}{l}\text { Adjuvant therapy } \\
\text { Yes } \\
\text { No }\end{array}$ & 2.033 & 0.154 & & NI \\
\hline
\end{tabular}

ignored genes in the 10-gene signature may become potential novel markers for NSCLC. However, the present study also has certain limitations. First, the study consisted solely of bioinformatics research, and there was no validation of clinical sample data. Second, the study only verified the validity and reliability of the 10-gene signature impact on the overall survival of the patients, but did not verify relapse-free survival. Third, the sample size requires further expansion to verify the accuracy of the 10-gene signature and truly clarify its clinical value.

\section{Acknowledgements}

Not applicable.

\section{Funding}

This study was supported jointly by Special funds for Taishan Scholars Project (grant. no. tsqn201812149), Academic promotion programme of Shandong First Medical University (2019RC004). 


\section{Availability of data and material}

We declared that materials described in the manuscript, including all relevant raw data, are freely available to any scientist wishing to use them for non-commercial purposes, without breaching participant confidentiality.

\section{Authors' contributions}

HW designed the project and proposed the research concept. $\mathrm{XL}$ carried out the data search and downloading and literature collection. CZ conducted the bioinformatic analysis and CZ constructed the graphic images and data charts and performed the statistical processing. JL wrote the manuscript. All authors read and approved the manuscript and agree to be accountable for all aspects of the research in ensuring that the accuracy or integrity of any part of the work are appropriately investigated and resolved.

\section{Ethics approval and consent to participate}

This study was approved by the Ethics Committee of Shandong Cancer Hospital and Institute and was consistent with the Helsinki Declaration. This study was mainly based on the Gene Expression Omnibus datasets (GEO; GSE103584,GSE31210) and The Cancer Genome Atlas (TCGA, https://tcga-data.nci. nih.gov/tcga/). Personal privacy information was not involved, thus informed consent was not needed.

\section{Patient consent for publication}

Not applicable.

\section{Competing interests}

The authors declare that they have no competing interests.

\section{References}

1. Bray F, Ferlay J, Soerjomataram I, Siegel RL, Torre LA and Jemal A: Global cancer statistics 2018: GLOBOCAN estimates of incidence and mortality worldwide for 36 cancers in 185 countries. CA Cancer J Clin 68: 394-424, 2018.

2. Song P, Cui X, Bai L, Zhou X, Zhu X, Zhang J, Jin F, Zhao J, Zhou C, Zhou Y, et al: Molecular characterization of clinical responses to $\mathrm{PD}-1 / \mathrm{PD}-\mathrm{L} 1$ inhibitors in non-small cell lung cancer: Predictive value of multidimensional immunomarker detection for the efficacy of PD-1 inhibitors in Chinese patients. Thorac Cancer 10: 1303-1309, 2019.

3. Zhang C, Leighl NB, Wu YL and Zhong WZ: Emerging therapies for non-small cell lung cancer. J Hematol Oncol 12: 45, 2019.

4. Chansky K, Sculier JP, Crowley JJ, Giroux D, Van Meerbeeck J and Goldstraw P; International Staging Committee and Participating Institutions: The international association for the study of lung cancer staging project: Prognostic factors and pathologic TNM stage in surgically managed non-small cell lung cancer. J Thorac Oncol 4: 792-801, 2009.

5. Goldstraw P, Ball D, Jett JR, Le Chevalier T, Lim E, Nicholson AG and Shepherd FA: Non-small-cell lung cancer. Lancet 378: 1727-1740, 2011.

6. Hanahan D and Weinberg RA: The hallmarks of cancer. Cell 100: 57-70, 2000.

7. Hanahan D and Coussens LM: Accessories to the crime: Functions of cells recruited to the tumor microenvironment. Cancer Cell 21: 309-322, 2012.
8. Stankovic B, Bjørhovde HA, Skarshaug R, Aamodt H, Frafjord A, Müller E, Hammarström C, Beraki K, Bækkevold ES, Woldbæk PR, et al: Immune cell composition in human non-small cell lung cancer. Front Immunol 9: 3101, 2019.

9. Galon J, Pagès F, Marincola FM, Angell HK, Thurin M, Lugli A, Zlobec I, Berger A, Bifulco C, Botti G, et al: Cancer classification using the immunoscore: A worldwide task force. J Transl Med 10: 205, 2012.

10. Xiong Y, Liu L, Xia Y, Qi Y, Chen Y, Chen L, Zhang P, Kong Y, $\mathrm{Qu} Y$, Wang Z, et al: Tumor infiltrating mast cells determine oncogenic HIF- $2 \alpha$-conferred immune evasion in clear cell renal cell carcinoma. Cancer Immunol Immunother 68: 731-741, 2019.

11. Rohr-Udilova N, Klinglmüller F, Schulte-Hermann R, Stift J, Herac M, Salzmann M, Finotello F, Timelthaler G, Oberhuber G, Pinter M, et al: Deviations of the immune cell landscape between healthy liver and hepatocellular carcinoma. Sci Rep 8: 6220, 2018.

12. Chen B, Khodadoust MS, Liu CL, Newman AM and Alizadeh AA: Profiling tumor infiltrating immune cells with CIBERSORT. Methods Mol Biol 1711: 243-259, 2018.

13. Yoshihara K, Shahmoradgoli M, Martínez E, Vegesna R, Kim H, Torres-Garcia W, Treviño V, Shen H, Laird PW, Levine DA, et al: Inferring tumour purity and stromal and immune cell admixture from expression data. Nat Commun 4: 2612, 2013.

14. Bakr S, Gevaert O, Echegaray S, Ayers K, Zhou M, Shafiq M, Zheng $\mathrm{H}$, Benson JA, Zhang W, Leung ANC, et al: A radiogenomic dataset of non-small cell lung cancer. Sci Data 5: 180202, 2018.

15. Okayama H, Kohno T, Ishii Y, Shimada Y, Shiraishi K, Iwakawa R, Furuta K, Tsuta K, Shibata T, Yamamoto S, et al: Identification of genes upregulated in ALK-positive and EGFR/KRAS/ALK-negative lung adenocarcinomas. Cancer Res 72: 100-111, 2012.

16. Camp RL, Dolled-Filhart M and Rimm DL: X-tile: A new bio-informatics tool for biomarker assessment and outcomebased cut-point optimization. Clin Cancer Res 10: 7252-7259, 2004.

17. Ritchie ME, Phipson B, Wu D, Hu Y, Law CW, Shi W and Smyth GK: Limma powers differential expression analyses for RNA-sequencing and microarray studies. Nucleic Acids Res 43: e47, 2015 .

18. Huang da W, Sherman BT and Lempicki RA: Systematic and integrative analysis of large gene lists using DAVID bioinformatics resources. Nat Protoc 4: 44-57, 2009.

19. Tibshirani R: The lasso method for variable selection in the Cox model. Stat Med 16: 385-395, 1997.

20. Friedman J, Hastie T and Tibshirani R: Regularization paths for generalized linear models via coordinate descent. J Stat Softw 33: 1-22, 2010.

21. Newman AM, Liu CL, Green MR, Gentles AJ, Feng W, Xu Y, Hoang CD, Diehn M and Alizadeh AA: Robust enumeration of cell subsets from tissue expression profiles. Nat Methods 12: 453-457, 2015.

22. O'Quigley J and Moreau T: Cox's regression model: Computing a goodness of fit statistic. Comput Methods Programs Biomed 22: 253-256, 1986.

23. Heagerty PJ, Lumley T and Pepe MS: Time-dependent ROC curves for censored survival data and a diagnostic marker. Biometrics 56: 337-344, 2000.

24. Fridman WH, Pagès F, Sautès-Fridman $\mathrm{C}$ and Galon $\mathrm{J}$ : The immune contexture in human tumours: Impact on clinical outcome. Nat Rev Cancer 12: 298-306, 2012.

25. Wojas-Krawczyk K, Kalinka E, Grenda A, Krawczyk P and Milanowski J: Beyond PD-L1 markers for lung cancer immunotherapy. Int J Mol Sci 20: E1915, 2019.

26. Binnewies M, Roberts EW, Kersten K, Chan V, Fearon DF, Merad M, Coussens LM, Gabrilovich DI, Ostrand-Rosenberg S, Hedrick CC, et al: Understanding the tumor immune microenvironment (TIME) for effective therapy. Nat Med 24: 541-550, 2018.

27. Kilic A, Landreneau RJ, Luketich JD, Pennathur A and Schuchert MJ: Density of tumor-infiltrating lymphocytes correlates with disease recurrence and survival in patients with large non-small-cell lung cancer tumors. J Surg Res 167: 207-210, 2011.

28. Horne ZD, Jack R, Gray ZT, Siegfried JM, Wilson DO, Yousem SA, Nason KS, Landreneau RJ, Luketich JD and Schuchert MJ: Increased levels of tumor-infiltrating lymphocytes are associated with improved recurrence-free survival in stage 1A non-small-cell lung cancer. J Surg Res 171: 1-5, 2011. 
29. Domagala-Kulawik J: The role of the immune system in non-small cell lung carcinoma and potential for therapeutic intervention. Transl Lung Cancer Res 4: 177-190, 2015.

30. Pabla S, Conroy JM, Nesline MK, Glenn ST, Papanicolau-Sengos A, Burgher B, Hagen J, Giamo V, Andreas J, Lenzo FL, et al: Proliferative potential and resistance to immune checkpoint blockade in lung cancer patients. J Immunother Cancer 7: 27, 2019.

31. Shin DS, Zaretsky JM, Escuin-Ordinas H, Garcia-Diaz A Hu-Lieskovan S, Kalbasi A, Grasso CS, Hugo W, Sandoval S, Torrejon DY, et al: Primary resistance to PD-1 blockade mediated by JAK1/2 mutations. Cancer Discov 7: 188-201, 2017.

32. Kato S, Goodman A, Walavalkar V, Barkauskas DA, Sharabi A and Kurzrock R: Hyperprogressors after immunotherapy: Analysis of genomic alterations associated with accelerated growth rate. Clin Cancer Res 23: 4242-4250, 2017.

33. Champiat S, Dercle L, Ammari S, Massard C, Hollebecque A, Postel-Vinay S, Chaput N, Eggermont A, Marabelle A, Soria JC and Ferté C: Hyperprogressive disease is a new pattern of progression in cancer patients treated by anti-PD-1/PD-L1. Clin Cancer Res 23: 1920-1928, 2017.

34. Paoloni-Giacobino A, Chen $\mathrm{H}$ and Antonarakis SE: Cloning of a novel human neural cell adhesion molecule gene (NCAM2) that maps to chromosome region 21q21 and is potentially involved in Down syndrome. Genomics 43: 43-51, 1997.

35. Rasmussen KK, Falkesgaard MH, Winther M, Roed NK, Quistgaard CL, Teisen MN,Edslev SM, Petersen DL, Aljubouri A, Christensen C, et al: NCAM2 Fibronectin type-III domains form a rigid structure that binds and activates the Fibroblast Growth Factor Receptor. Sci Rep 8: 8957, 2018.

36. Edwards S, Campbell C, Flohr P, Shipley J, Giddings I, Te-Poele R, Dodson A, Foster C, Clark J, Jhavar S, et al: Expression analysis onto microarrays of randomly selected cDNA clones highlights HOXB13 as a marker of human prostate cancer. Br J Cancer 92 376-381, 2005

37. Romanuik TL, Ueda T, Le N, Haile S, Yong TM, Thomson T, Vessella RL and Sadar MD: Novel biomarkers for prostate cancer including noncoding transcripts. Am J Pathol 175: 2264-2276, 2009

38. von der Heyde S, Wagner S, Czerny A, Nietert M, Ludewig F, Salinas-Riester G, Arlt D and Beißbarth T: mRNA profiling reveals determinants of trastuzumab efficiency in HER2-positive breast cancer. PLoS One 10: e0117818, 2015.

39. Lim B, Park JL, Kim HJ, Park YK, Kim JH, Sohn HA, Noh SM, Song KS, Kim WH, Kim YS and Kim SY: Integrative genomics analysis reveals the multilevel dysregulation and oncogenic characteristics of TEAD4 in gastric cancer. Carcinogenesis 35: 1020-1027, 2014

40. Mayanagi T, Morita T, Hayashi K, Fukumoto K and Sobue K: Glucocorticoid receptor-mediated expression of caldesmon regulates cell migration via the reorganization of the actin cytoskeleton. J Biol Chem 283: 31183-31196, 2008.

41. Thorsen K, Sørensen KD, Brems-Eskildsen AS, Modin C, Gaustadnes M, Hein AM, Kruhøffer M, Laurberg S, Borre M, Wang K, et al: Alternative splicing in colon, bladder, and prostate cancer identified by exon array analysis. Mol Cell Proteomics 7: 1214-1224, 2008.

42. Liu J, Li H, Shen S, Sun L, Yuan Y and Xing C: Alternative splicing events implicated in carcinogenesis and prognosis of colorectal cancer. J Cancer 9: 1754-1764, 2018.

43. Liu Y, Wu X, Wang G, Hu S, Zhang Y and Zhao S: CALD1, CNN1, and TAGLN identified as potential prognostic molecular markers of bladder cancer by bioinformatics analysis. Medicine (Baltimore) 98: e13847, 2019.

44. Chauvin A, Wang CS, Geha S, Garde-Granger P, Mathieu AA, Lacasse V and Boisvert FM: The response to neoadjuvan chemoradiotherapy with 5-fluorouracil in locally advanced rectal cancer patients: A predictive proteomic signature. Clin Proteomics 15: 16, 2018.

45. Yokota M, Kojima M, Higuchi Y, Nishizawa Y, Kobayashi A, Ito M, Saito N and Ochiai A: Gene expression profile in the activation of subperitoneal fibroblasts reflects prognosis of patients with colon cancer. Int J Cancer 138: 1422-1431, 2016.

46. Wood LD, Parsons DW, Jones S, Lin J, Sjöblom T, Leary RJ, Shen D, Boca SM, Barber T, Ptak J, et al: The genomic landscapes of human breast and colorectal cancers. Science 318: 1108-1113, 2007

47. Coy JF, Wiemann S, Bechmann I, Bächner D, Nitsch R, Kretz O, Christiansen $\mathrm{H}$ and Poustka A: Pore membrane and/or filament interacting like protein 1 (POMFIL1) is predominantly expressed in the nervous system and encodes different protein isoforms. Gene 290: 73-94, 2002.
48. Soon PS, Gill AJ, Benn DE, Clarkson A, Robinson BG, McDonald KL and Sidhu SB: Microarray gene expression and immunohistochemistry analyses of adrenocortical tumors identify IGF2 and Ki-67 as useful in differentiating carcinomas from adenomas. Endocr Relat Cancer 16: 573-583, 2009.

49. Bleeker FE, Lamba S, Rodolfo M, Scarpa A, Leenstra S, Vandertop WP and Bardelli A: Mutational profiling of cancer candidate genes in glioblastoma, melanoma and pancreatic carcinoma reveals a snapshot of their genomic landscapes. Hum Mutat 30: E451-E459, 2009.

50. Hahtola S, Burghart E, Puputti M, Karenko L, Abdel-Rahman WM, VäkeväL,Jeskanen L, Virolainen S, Karvonen J,SalmenkiviK, et al: Cutaneous T-cell lymphoma-associated lung cancers show chromosomal aberrations differing from primary lung cancer. Genes Chromosomes Cancer 47: 107-117, 2008.

51. Karenko L, Hahtola S, Päivinen S, Karhu R, Syrjä S, Kähkönen M, Nedoszytko B, Kytölä S, Zhou Y, Blazevic V, et al: Primary cutaneous T-cell lymphomas show a deletion or translocation affecting NAV3, the human UNC-53 homologue. Cancer Res 65 : 8101-8110, 2005 .

52. Hahtola S, Burghart E, Jeskanen L, Karenko L, AbdelRahman WM, Polzer B, Kajanti M, Peltomäki P, Pettersson T, Klein CA and Ranki A: Clinicopathological characterization and genomic aberrations in subcutaneous panniculitis-like T-cell lymphoma. J Invest Dermatol 128: 2304-2309, 2008.

53. Zhang SD, McCrudden CM, Meng C, Lin Y and Kwok HF: The significance of combining VEGFA, FLT1, and KDR expressions in colon cancer patient prognosis and predicting response to bevacizumab. Onco Targets Ther 8: 835-843, 2015.

54. Kershaw MH, Westwood JA, Zhu Z, Witte L, Libutti SK and Hwu P: Generation of gene-modified $T$ cells reactive against the angiogenic kinase insert domain-containing receptor (KDR) found on tumor vasculature. Hum Gene Ther 11: 2445-2452, 2000.

55. Edelbauer M, Datta D, Vos IH, Basu A, Stack MP, Reinders ME, Sho M, Calzadilla K, Ganz P and Briscoe DM: Effect of vascular endothelial growth factor and its receptor KDR on the transendothelial migration and local trafficking of human $\mathrm{T}$ cells in vitro and in vivo. Blood 116: 1980-1989, 2010.

56. He J, Xia M, Tsang WH, Chow KL and Xia J: ICA1L forms BAR-domain complexes with PICK1 and is crucial for acrosome formation in spermiogenesis. J Cell Sci 128: 3822-3836, 2015.

57. Parvin S, Rezazadeh M, Hosseinzadeh H, Moradi M, Shiva S and Gharesouran J: The neuronal ceroid lipofuscinoses-linked loss of function CLN5 and CLN8 variants disrupt normal lysosomal function. Neuromolecular Med 21: 160-169, 2019.

58. Qi J, Zhang X, Zhang HK, Yang HM, Zhou YB and Han ZG: ZBTB34, a novel human BTB/POZ zinc finger protein, is a potential transcriptional repressor. Mol Cell Biochem 290: 159-167, 2006

59. Clemens RA, Newbrough SA, Chung EY, Gheith S, Singer AL, Koretzky GA and Peterson EJ: PRAM-1 is required for optimal integrin-dependent neutrophil function. Mol Cell Biol 24: 10923-10932, 2004

60. Moog-Lutz C, Peterson EJ, Lutz PG, Eliason S, Cavé-Riant F, Singer A, Di Gioia Y, Dmowski S, Kamens J, Cayre YE and Koretzky G: PRAM-1 is a novel adaptor protein regulated by retinoic acid (RA) and promyelocytic leukemia (PML)-RA receptor alpha in acute promyelocytic leukemia cells. J Biol Chem 276: 22375-22381, 2001.

61. Gill RM, Michael A, Westley L, Kocher HM, Murphy JI and Dhoot GK: SULF1/SULF2 splice variants differentially regulate pancreatic tumour growth progression. Exp Cell Res 324: 157-171, 2014.

62. Morimoto-Tomita M, Uchimura K, Werb Z, Hemmerich S and Rosen SD: Cloning and characterization of two extracellular heparin-degrading endosulfatases in mice and humans. J Biol Chem 277: 49175-49185, 2002

63. Hur K, Han TS, Jung EJ, Yu J, Lee HJ, Kim WH, Goel A and Yang HK: Up-regulated expression of sulfatases (SULF1 and SULF2) as prognostic and metastasis predictive markers in human gastric cancer. J Pathol 228: 88-98, 2012.

This work is licensed under a Creative Commons Attribution-NonCommercial-NoDerivatives 4.0 International (CC BY-NC-ND 4.0) License. 